Oper Orthop Traumatol 2015 $\cdot 27: 100-100$

DOI 10.1007/s00064-014-0340-1

Online publiziert: 12. April 2015

c) Springer-Verlag Berlin Heidelberg 2015

\title{
T. Mittlmeier
}

Chirurgische Klinik und Poliklinik, Abteilung für Unfall-, Hand- und

Wiederherstellungschirurgie, Universitätsmedizin Rostock

\section{Operative}

\section{Rekonstruktionsverfahren beim Charcot-Fuß}

derlich sind, beeinflussen Lokalisation der Manifestation der DNOAP und deren individuelle Dynamik den Heilverlauf und die Prognose.

Mittlerweile koexistieren unterschiedliche chirurgische Interventionsstrategien und Stabilisationskonzepte, deren Evidenz nicht über das Level 4 bis 5 hinauskommt, da nach wie vor multizentrische prospektive randomisierte Studien fehlen. Allen Therapieverfahren bei DNOAP gemeinsam ist eine hohe peri- und postoperative Komplikationsrate, die $20-30 \%$ betragen kann.

Im vorliegenden Heft sollen mit den 4 Beiträgen zur chirurgisch-rekonstruktiven Intervention bei DNOAP an Rückund Mittfuß - den beiden Lokalisationen mit den höchsten Instabilitätsraten bei DNOAP - unter Berücksichtigung interner, externer und kombinierter Stabilisationstechniken der aktuelle Kenntnisstand und gangbare Wege für einen Extremitätenerhalt in Funktionsstellung und die Besserung der Lebensqualität der Betroffenen aufgezeigt werden. Dem mit der operativen Therapiewahl bei DNOAP befassten kundigen Arzt sollen diese Beiträge eine wertvolle Hilfe bei der individuellen Entscheidungsfindung leisten.

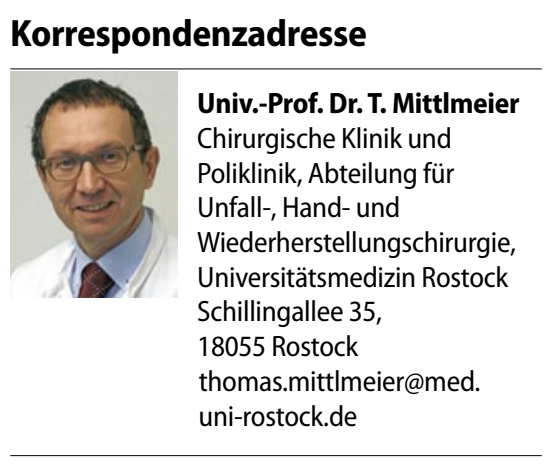

Einhaltung ethischer Richtlinien

Interessenkonflikt. T. Mittlmeier gibt an, dass kein Interessenkonflikt besteht.

\section{Literatur}

1. Rodgers $\mathrm{LC}$, Frykberg RG, Armstrong DG et al (2011) Consensus report: the Charcot foot in diabetes. Diabetes Care 34:2123-2129 